\title{
MOLECULAR ORBITAL STUDIES ON ELECTRONIC SPECTRA OF SOME SUBSTITUTED ANTHRAQUINONE CATION RADICALS
}

\author{
M. AhMed AND Z.H. KhaN* \\ Department of Physics, Jamia Millia Islamia \\ Jamia Nagar, New Delhi-110025, India \\ (Received January 3, 1995; revised version March 8, 1995)

\begin{abstract}
Electronic transition energies of radical cations of some hydroxy-substituted anthraquinones are calculated using an open-shell self-consistent field method with limited configuration interaction. The results are analyzed and a correlation diagram is given which provides useful information about the characteristic behaviour of the electronic transitions depending on the positions of the hydroxyl substituents. Also, the first ionization potentials of "substituted anthraquinones are calculated using an empirical relation connecting ionization potentials with the lowest-energy non-Koopmans bands for their radical cations. Such information is particularly useful for systems like substituted anthraquinones for which uv photoelectron spectroscopic data are in scarce.
\end{abstract}

PACS numbers: $31.20 .-\mathrm{d}$

\section{Introduction}

Our interest in radical cations of hydroxy-substituted aromatics started with the publication of the electronic spectra of monopositive ions of 1- and 2-naphthols [1]. This was followed by a comprehensive theoretical study of dihydroxy naphthalenes $[2,3]$ where it was demonstrated that, even in the absence of experimental data, open-shell SCF-CI (self-consistent field configuration interaction) calculations lead to some very useful information about energies and intensities of electronic transitions. The present paper on radical cations of anthraquinones is an extension of the afore-mentioned work. Anthraquinones as well as their hydroxy derivatives are of wide interest in a variety of fields. For instance, some of them provide a model for chromophores of many substances of biochemical and pharmaceutical interest [4]. Some anthraquinones also serve to metallize semiconductor

\footnotetext{
*Author to whom correspondence should be sent.
} 
surfaces due to their peculiar redox behaviour [5] whereas others are important in the production of dyes and intermediates [6].

In the past, we have successfully produced radical cations of polycyclic aromatic hydrocarbons in boric acid matrix. But for hydroxy-substituted aromatics, the situation is rather complex primarily due to hydrogen bonding between the solute and the boric acid and also due to some complex formation [7]. Another difficulty with such molecular systems lies in their possible decomposition on heating which is perhaps the reason that uv photoelectron spectroscopic (PES) data on such species are not readily a vailable.

In the absence of experimental data on electronic spectra of radical cations of hydroxy-anthraquinones, we have made a detailed theoretical study on the series, 1.2-, 1.4-, 1.5-, 1.8-dihydroxy 9.10-anthraquinones, and 1.2,5.8-tetrahydroxy 9.10-anthraquinone. Their electronic transition energies and intensities are calculated using open-shell SCF-CI methods. Also, we have presented a correlation diagram and have examined in detail the variation in energies and intensities of various electronic transitions as a function of hydroxyl group substitution at differcnt atomic positions in the ionic systems. We have further estimated the first ionization potentials (IPs) of the hydroxy-anthraquinones from the calculated energies of the lowest non-Koopmans states of their radical cations.

\section{Calculations}

For the calculation of electronic transition energies and intensities for the hydroxy-anthraquinones, we have used two different theoretical models: LonguetHiggins and Pople (LIIP) method [8] and Wasilewski method [9]. Considering that the ground state configuration of an open-shell system with an odd number of $\pi$-electrons can be written in the form

$$
{ }^{2} \Psi_{\mathrm{g}}=\left|\Phi_{i} \bar{\Phi}_{i} \ldots \Phi_{k} \bar{\Phi}_{k} \ldots \Phi_{m}\right|,
$$

where the indices $k$ and $m$ correspond to the doubly-occupied and singly-occupied molecular orbitals (MOs), respectively, we can form different types of excited state doublet configurations from the $\pi$ one-electron excitations. These may be classified as $\mathrm{I}(k \rightarrow m), \mathrm{A}(m \rightarrow m+1), \mathrm{B}_{1}(m \rightarrow x ; x>m+1)$, and $\mathrm{B}_{2}, \mathrm{~B}_{3}(k \rightarrow x)$, where the letter $x$ represents vacant MOs. Other theoretical details are given in Ref. $[2,3]$. The Coulomb and resonance integrals for heteroatoms are calculated as before from the expressions

$$
\begin{aligned}
& \alpha_{\mathrm{O}}=\alpha_{\mathrm{C}}+h_{\mathrm{O}} \beta_{\mathrm{CC}}, \\
& \beta_{\mathrm{CO}}=k_{\mathrm{CO}} \beta_{\mathrm{CC}},
\end{aligned}
$$

where $\alpha_{\mathrm{C}}$ and $\beta_{\mathrm{CC}}$ are the Coulomb and resonance integrals for unsubstituted systems and the subscripts $\mathrm{O}$ and $\mathrm{C}$ are used for oxygen and carbon atoms, respectively. Also, we choose $h_{\mathrm{O}}=2.0$ and $k_{\mathrm{CO}}=0.8$. Furthermore, we have taken $\beta_{\mathrm{CC}}=-2.27 \mathrm{eV}$ and $\beta_{\mathrm{CO}}=-2.30 \mathrm{eV}$ for bonded atoms, whereas for non-bonded atoms the resonance integrals are neglected. The one-centre Coulomb integrals are evaluated by taking the difference of ionization potentials $\left(I_{\mathrm{C}}\right.$ or $\left.I_{\mathrm{O}}\right)$ and electron affinities $\left(A_{\mathrm{C}}\right.$ or $\left.A_{\mathrm{O}}\right)$. The two-centre integrals are computed using the Mataga-Nishimoto approximation [10]. For ionization potentials and electron 
affinities, the following parameters are chosen: $I_{\mathrm{C}}=11.16 \mathrm{eV}, I_{\mathrm{O}}=32.9 \mathrm{eV}$, $A_{\mathrm{C}}=0.03 \mathrm{eV}$, and $A_{\mathrm{O}}=11.7 \mathrm{eV}$. Calculations were made using a software package "IONSP1" developed by one of the authors.[11] on a HCL Magnum Multi-Risc Computer in the Department of Physics of the University. Since the results obtained from both the LHP as well as Wasilewski methods are almost identical, we have adopted the former one in the following discussion.

\section{Results and discussion}

Results of open-shell calculations based on Longuet-Higgins and Pople method for anthraquinones radical cations are given in Tables I-V. The first column of each table represents the alphabetical enumeration of the calculated electronic transitions. The second column gives the calculated electronic transition energies in $\mathrm{eV}$ followed by their oscillator strengths $f$. A classification of electronic transitions is made on the basis of contributions from different configurations. When the contribution from the second major configuration is smaller than 0.3 , transitions are considered as "pure", whereas for contributions $\geq 0.3$, they are assumed to be "mixed". The pure transitions are generally represented by a one-letter symbol, but for simplicity only two-letter symbols are chosen for the mixed transitions. The character of a particular state is determined on the basis of contributions from various major configurations given in the last three columns along with their classes.

\subsection{4-dihydroxy 9.10-anthraquinone cation [1.4-DHAQ+]}

The calculated electronic transition energies and intensities for 1.4-DHAQ cation are collected in Table I. This molecular system has $C_{2 v}$ symmetry as a result of which all of its electronic transitions are polarized either along its longer axis $(x)$ or the shorter axis $(y)$. The first two electronic transitions ( $a$ and $b$ ) of 1.4-DIIAQ cation are of IB-type having mixed character. Similar is the case with the $q, t$, and $u$ transitions. The transitions $c, d$, and $v$ are of I-type with the middle one having a larger $f$-value. The sixth electronic transition $f$ is weak and is assigned as IA which results due to the interaction of three configurations $\Phi_{4} \rightarrow \Phi_{10}, \Phi_{9} \rightarrow \Phi_{10}$ and $\Phi_{10} \rightarrow \Phi_{11}$. The mixing of the above configurations also gives rise to a higher energy component AI located at $3.76 \mathrm{eV}$ with a moderate oscillator strength. Calculations predict an intense transition $h$ of BA type which arises due to the mixing of the configurations $\Phi_{9} \rightarrow \Phi_{11}$ and $\Phi_{10} \rightarrow \Phi_{11}$. The MO diagram for 1.4-DHAQ ${ }^{+}$showing all the above transitions is depicted in Fig. 1.

\subsection{8-dihydroxy 9.10-anthraquinone cation [1.8-DHAQ $\left.Q^{+}\right]$}

Calculated energies and the intensities for electronic transitions in 1.8-DHAQ cation are given in Table II and a sketch of its MOs along with the transitions is shown in Fig. 2. This molecular system is also of $\mathrm{C}_{2 v}$ symmetry, thus its electronic transitions are either $x$-polarized or $y$-polarized. The first four transitions $(a, b, c$, and $d$ ) in the cation are pure I-type one-electron excitations all having very small oscillator strength. The transitions $f, k, v$, and $x$ are of IB type. 

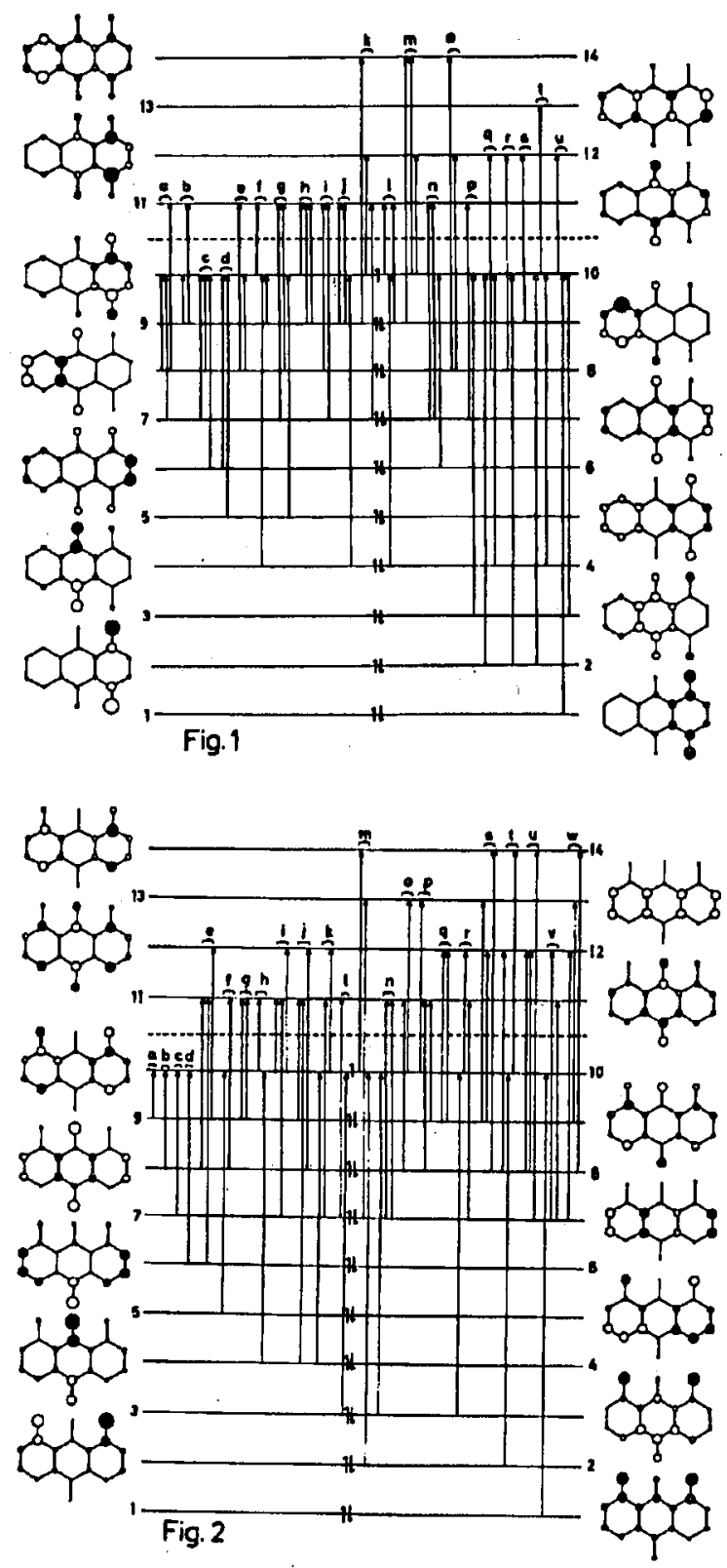

Fig. 1. MO diagram for 1.4-dihydroxy 9.10-anthraquinone cation with different modes of excitation. The horizontal broken line separates the singly-occupied orbital from the lowest vacant orbital. The vertical lines with arrows represent the major configurations whose coefficient in the total wave function exceeds 0.3 . The letters $a, b, c$ etc. correspond to the transitions in Table I

Fig. 2 MO diagram for 1.8-dihydroxy 9.10-anthraquinone cation and its different modes of excitation. The letters $a, b, c$ etc. correspond to the transitions in Table II. For other details, see caption to Fig. 1. 
TABLE I

Results of open-shell SCF-CI calculations for 1.4-dihydroxy 9.10-anthraquinone cation.

\begin{tabular}{|c|c|c|c|c|c|c|}
\hline & $E[\mathrm{eV}]$ & $f^{*}$ & Class & & Character ** & \\
\hline$a$ & 0.72 & $0.009(y)$ & IB & $-0.62(8 \rightarrow 10) \mathrm{I}$ & $-0.60(7 \rightarrow 10) I$ & $0.38(8 \rightarrow 11) \mathrm{B}_{3}$ \\
\hline$b$ & 1.18 & $0.037(x)$ & IB & $0.84(9 \rightarrow 10) \mathrm{I}$ & $-0.44(9 \rightarrow 11) \mathrm{B}_{3}$ & \\
\hline$c$ & 1.34 & $0.002(y)$ & I & $0.69(7 \rightarrow 10) \mathrm{I}$ & $-0.51(8 \rightarrow 10) \mathrm{I}$ & $-0.40(6 \rightarrow 10) \mathrm{I}$ \\
\hline$d$ & 1.85 & $0.058(y)$ & $\mathbf{I}$ & $0.61(6 \rightarrow 10) I$, & $0.57(5 \rightarrow 10) \mathrm{I}$ & \\
\hline$e$ & 2.62 & $0.034(y)$ & BI & $-0.56(8 \rightarrow 11) \mathrm{B}_{3}$, & $-0.48(8 \rightarrow 10) \mathrm{I}$ & \\
\hline$f$ & 2.73 & $0.001(x)$ & IA & $-0.48(10 \rightarrow 11) A$ & $0.46(4 \rightarrow 10) \mathrm{I}$ & $0.40(9 \rightarrow 10) \mathrm{I}$ \\
\hline$g$ & 3.07 & $0.005(y)$ & BI & $-v .46(7 \rightarrow 11) \mathrm{B}_{3}$ & $0.41(8 \rightarrow 11) B_{3}$ & $-0.39(5 \rightarrow 10) \mathrm{I}$ \\
\hline$h$ & 3.20 & $0.209(x)$ & $\mathrm{BA}$ & $-0.54(10 \rightarrow 11) A$ & $-0.53(9 \rightarrow 11) \mathrm{B}_{3}$ & $0.50(9 \rightarrow 11) \mathrm{B}_{2}$ \\
\hline$i$ & 3.46 & $0.088(y)$ & $\mathrm{B}$ & $-0.76(8 \rightarrow 11) \mathrm{B}_{2}$ & $0.37(7 \rightarrow 11) B_{2}$ & \\
\hline$j$ & 3.53 & $0.138(x)$ & BI & $0.70(9 \rightarrow 11) \mathrm{B}_{2}$ & $0.42(9 \rightarrow 11) \mathrm{B}_{3}$ & $-0.33(4 \rightarrow 10) \mathrm{I}$ \\
\hline$k$ & 3.53 & $0.001(y)$ & B & $0.58(9 \rightarrow 14) \mathrm{B}_{3}$ & $-0.45(10 \rightarrow 12) \mathrm{B}_{1}$ & $0.36(7 \rightarrow 11) \mathrm{B}_{3}$ \\
\hline$l$ & 3.76 & $0.057(x)$ & AI & $-0.58(10 \rightarrow 11) A$ & $-0.54(4 \rightarrow 10) I$ & $-0.40(9 \rightarrow 11) \mathrm{B}_{2}$ \\
\hline$m$ & 4.03 & $0.012(y)$ & B & $-0.39(9 \rightarrow 14) \mathrm{B}_{3}$ & $-0.32(10 \rightarrow 14) \mathrm{B}_{1}$ & $-0.32(10 \rightarrow 12) \mathrm{B}_{1}$ \\
\hline$n$ & 4.39 & $0.084(y)$ & BI & $0.57(7 \rightarrow 11) \mathrm{B}_{2}$ & $0.40(7 \rightarrow 11) \mathrm{B}_{3}$ & $0.33(6 \rightarrow 10) \mathrm{I}$ \\
\hline$o$ & 4.52 & $0.006(x)$ & B & $0.69(8 \rightarrow 14) \mathrm{B}_{3}$ & $-0.51(8 \rightarrow 12) \mathrm{B}_{3}$ & \\
\hline$p$ & 4.62 & $0.123(y)$ & BI & $-0.51(7 \rightarrow 11) \mathrm{B}_{2}$ & $0.30(3 \rightarrow 10) \mathrm{I}$ & \\
\hline$q$ & 4.63 & $0.000(x)$ & IB & $-0.50(2 \rightarrow 10) \mathrm{I}$ & $0.38(8 \rightarrow 12) B_{2}$ & $0.37(4 \rightarrow 10) \mathrm{I}$ \\
\hline$r$ & 4.85 & $0.208(x)$ & BI & $-0.68(8 \rightarrow 12) \mathrm{B}_{2}$ & $-0.43(2 \rightarrow 10) \mathrm{I}$ & \\
\hline$s$ & 4.92 & $0.005(y)$ & B & $0.88(9 \rightarrow 12) \mathrm{B}_{2}$ & & \\
\hline$t$ & 5.00 & $0.039(x)$ & IB & $-0.59(2 \rightarrow 10) \mathrm{I}$ & $-0.44(10 \rightarrow 13) \mathrm{B}_{1}$ & $-0.35(4 \rightarrow 10) \mathrm{I}$ \\
\hline$u$ & 5.04 & $0.564(y)$ & IB & $0.50(10 \rightarrow 12) \mathrm{B}_{1}$ & $-0.37(1 \rightarrow 10) \mathrm{I}$ & $-0.35(3 \rightarrow 10) \mathrm{I}$ \\
\hline$v$ & 5.11 & $0.027(y)$ & I & $-0.63(3 \rightarrow 10) I$ & $-0.33(5 \rightarrow 10) \mathrm{I}$ & \\
\hline$w$ & 5.14 & $0.215(x)$ & B & $-0.53(7 \rightarrow 12) \mathrm{B}_{2}$ & $-0.43(8 \rightarrow 14) \mathrm{B}_{2}$ & $-0.41(9 \rightarrow 13) \mathrm{B}_{2}$ \\
\hline$x$ & 5.24 & $0.220(x)$ & B & $-0.57(7 \rightarrow 12) \mathrm{B}_{2}$ & $0.39(9 \rightarrow 13) \mathrm{B}_{2}$ & $0.34(8 \rightarrow 14) \mathrm{B}_{2}$ \\
\hline$y$ & 5.54 & $0.119(y)$ & B & $-0.39(8 \rightarrow 13) B_{3}$ & $0.36(8 \rightarrow 13) \mathrm{B}_{2}$ & $-0.34(7 \rightarrow 11) \mathrm{B}_{3}$ \\
\hline$z$ & 5.61 & $0.209(y)$ & $\mathbf{B}$ & $0.85(6 \rightarrow 11) B_{2}$ & & \\
\hline
\end{tabular}

The mixing of the configurations $\Phi_{10} \rightarrow \Phi_{11}$ and $\Phi_{4} \rightarrow \Phi_{10}$ gives rise to the non-Koopmans AI-type transition ( $x$-polarized). The next transition $i$ is also of non-Koopmans type and belongs to the class $A B$, but it is far more intense than its predecessor. The transition $i$ results from the interaction of the configurations $\Phi_{10} \rightarrow \Phi_{11}, \Phi_{7} \rightarrow \Phi_{11}$ and $\Phi_{10} \rightarrow \Phi_{12}$. Mixing of the configurations $\Phi_{7} \rightarrow \Phi_{11}$ and $\Phi_{3} \rightarrow \Phi_{10}$ gives rise to the transitions $l$ and $n$ which are identified as BI $(x$-polarized). Calculations reveal that the most intense transition $t$ is located at $5.02 \mathrm{eV}$ which is polarized along the shorter molecular axis.

\subsection{5-dihydroxy 9.10-anthraquinone cation [1.5-DHAQ $\left.Q^{+}\right]$}

In Table III we have listed the results of calculations for 1.5-DHAQ cation where the first four transitions $(a, b, c$, and $d)$ are found to be pure I-type one-electron excitations. The other I-type transitions have mixed character. Of these, except for the transitions $j, t$, and $x$, all other have poor oscillator strengths. 
TABLE II

Results of open-shell SCF-CI calculations for 1.8-dihydroxy 9.10-anthraquinone cation.

\begin{tabular}{|c|c|c|c|c|c|c|}
\hline & $E[\mathrm{eV}]$ & $f^{*}$ & Class & & Character ${ }^{*}$ & \\
\hline$a$ & 0.16 & $0.032(x)$ & I & $0.96(9 \rightarrow 10) \mathrm{I}$ & & \\
\hline$b$ & 0.69 & $0.000(x)$ & I & $-0.98(8 \rightarrow 10) \mathrm{I}$ & & \\
\hline$c$ & 0.90 & $0.000(y)$ & I & $0.98(7 \rightarrow 10) I$ & + & \\
\hline$d$ & 2.06 & $0.000(x)$ & I & $0.87(6 \rightarrow 10) I$ & & \\
\hline$e$ & 2.21 & $0.016(y)$ & B & $-0.78(8 \rightarrow 11) \mathrm{B}_{3}$ & $-0.33(6 \rightarrow 11) \dot{B}_{3}$ & $-0.32(9 \rightarrow 12) \mathbf{B}_{3}$ \\
\hline$f$ & 2.38 & $0.036(y)$ & IB & $0.81(5 \rightarrow 10) \mathrm{I}$ & $-0.34(8 \rightarrow 11) \mathrm{B}_{3}$ & \\
\hline$g$ & 2.64 & $0.005(y)$ & B & $0.61(9 \rightarrow 11) \mathrm{B}_{3}$ & $0.53(9 \rightarrow 11) \mathrm{B}_{2}$ & \\
\hline$h$ & 3.24 & $0.015(x)$ & AI & $-0.63(10 \rightarrow 11) \mathrm{A}$ & $0.59(4 \rightarrow 10) \mathrm{I}$ & \\
\hline$i$ & 3.38 & $0.118(x)$ & $\mathbf{A B}$ & $-0.62(10 \rightarrow 11) \mathrm{A}$ & $0.41(7 \rightarrow 11) \mathrm{B}_{3}$ & $0.33(10 \rightarrow 12) \mathrm{B}_{1}$ \\
\hline$j$ & 3.42 & $0.001(y)$ & B & $-0.71(9 \rightarrow 11) \mathrm{B}_{2}$ & $-0.46(4 \rightarrow 11) \mathrm{B}_{3}$ & $0.34(8 \rightarrow 12) \mathrm{B}_{3}$ \\
\hline$k$ & 3.46 & $0.098(x)$ & IB & $-0.72(4 \rightarrow 10) \mathrm{I}$ & $-0.36(7 \rightarrow 11) \mathrm{B}_{3}$ & $-0.31(10 \rightarrow 12) \mathrm{B}_{1}$ \\
\hline$l$ & 3.93 & $0.014(x)$ & BI & $-0.83(7 \rightarrow 11) \mathrm{B}_{2}$ & $-0.40(3 \rightarrow 10) I$ & \\
\hline$m$ & 3.98 & $0.004(y)$ & BI & $0.51(10 \rightarrow 14) \mathrm{B}_{1}$ & $0.42(7 \rightarrow 13) \mathrm{B}_{3}$ & $0.34(2 \rightarrow 10) I$ \\
\hline$n$ & 4.27 & $0.000(x)$ & BI & $-0.58(3 \rightarrow 10) \mathrm{I}$ & $0.44(7 \rightarrow 11) \mathrm{B}_{3}$ & $0.40(7 \rightarrow 11) B_{2}$ \\
\hline 0 & 4.45 & $0.180(y)$ & B & $0.68(8 \rightarrow 11) \mathrm{B}_{2}$ & $-0.34(10-13) \mathrm{B}_{1}$ & \\
\hline $\mathrm{p}$ & 4.48 & $0.083(y)$ & B & $-0.55(10 \rightarrow 13) \mathrm{B}_{1}$ & $-0.51(8 \rightarrow 11) \mathrm{B}_{2}$ & $0.32(9 \rightarrow 11) \mathrm{B}_{3}$ \\
\hline$q$ & 4.53 & $0.216(y)$ & B & $0.68(9 \rightarrow 12) \mathbf{B}_{2}$ & $0.40(9 \rightarrow 12) \mathrm{B}_{3}$ & \\
\hline$r$ & 4.66 & $0.022(x)$ & $\mathrm{BI}$ & $0.52(3 \rightarrow 10) \mathrm{I}$ & $-0.50(10 \rightarrow 12) \mathrm{B}_{1}$ & $0.31(7 \rightarrow 11) \mathrm{B}_{3}$ \\
\hline$s$ & 4.77 & $0.060(x)$ & B & $0.62(9 \rightarrow 13) \mathrm{B}_{2}$ & $0.38(7 \rightarrow 12) \mathrm{B}_{2}$ & $-0.33(8 \rightarrow 14) \mathrm{B}_{2}$ \\
\hline$t$ & 5.02 & $0.414(y)$ & $\mathrm{BI}$ & $0.41(8 \rightarrow 12) \mathrm{B}_{2}$ & $-0.40(2 \rightarrow 10) \mathrm{I}$ & $0.37(10 \rightarrow 14) \mathrm{B}_{1}$ \\
\hline$u$ & 5.15 & $0.151(y)$ & B & $0.42(8 \rightarrow 12) \mathrm{B}_{2}$ & $-0.39(8 \rightarrow 12) \mathrm{B}_{3}$ & $0.35(7 \rightarrow 14) \mathrm{B}_{3}$ \\
\hline$v$ & 5.32 & $0.073(x)$ & IB & $0.62(1 \rightarrow 10) \mathrm{I}$ & $-0.32(7 \rightarrow 12) \mathrm{B}_{3}$ & $-0.31(7 \rightarrow 11) \mathrm{B}_{3}$ \\
\hline$w$ & 5.38 & $0.195(x)$ & B & $0.49(7 \rightarrow 12) B_{3}$ & $0.46(9 \rightarrow 13) \mathrm{B}_{2}$ & $-0.38(8 \rightarrow 14) \mathrm{B}_{3}$ \\
\hline$x$ & 5.54 & $0.028(y)$ & IB & $-0.64(2 \rightarrow 10) \mathrm{I}$ & $0.37(6 \rightarrow 11) \mathrm{B}_{3}$ & \\
\hline$y$ & 5.62 & $0.333(x)$ & B & $-0.67(9 \rightarrow 14) \mathrm{B}_{2}$ & $-0.35(7 \rightarrow 12) \mathrm{B}_{2}$ & \\
\hline$z$ & 5.71 & $0.002(y)$ & $\mathrm{B}$ & $-0.52(8 \rightarrow 12) \mathrm{B}_{2}$ & $-0.50(8 \rightarrow 12) \mathrm{B}_{3}$ & $-0.32(6 \rightarrow 11) \mathrm{B}_{2}$ \\
\hline
\end{tabular}

The $e$ and $f$ transitions result due to strong interaction between the configurations $\Phi_{5} \rightarrow \Phi_{10}$ and $\Phi_{8} \rightarrow \Phi_{11}$ and are assigned as IB and BI, respectively with most of the intensity being confined in the former. The A-type transition $i$ is a pure one-electron excitation $\Phi_{10} \rightarrow \Phi_{11}$ of moderate intensity. The transitions $l$ and $n$ fall in the same class BI and result from the mixing of the configurations $\Phi_{7} \rightarrow \Phi_{11}$ and $\Phi_{3} \rightarrow \Phi_{10}$. The MO diagram along with different electronic transitions is depicted in Fig. 3.

\subsection{2-dihydroxy 9.10-anthraquinone calion [1.2-DHAQ $Q^{+}$]}

Figure 4 shows the MO energy levels and electronic transitions of 1.2-DHAQ cation as calculated from the open-shell SCF theory the results for which are collected in Table IV. Its electronic transitions $(a, b, c, d$, and $f)$ arise from the I-type one-electron excitations. The transition $a$ results from the mixing of configurations $\Phi_{7} \rightarrow \Phi_{10}$ and $\Phi_{8} \rightarrow \Phi_{10}$. The transition $b$ arises due to the interaction of $\Phi_{7} \rightarrow \Phi_{10}$, $\Phi_{8} \rightarrow \Phi_{10}$ and $\Phi_{6} \rightarrow \Phi_{10}$ configurations. Both of the above transitions are weak in intensity. The transition $c$ is a pure one-electron excitation $\Phi_{9} \rightarrow \Phi_{10}$. It has a relatively large oscillator strength. The next two I-type transitions $d$ and $f$ have dominant contributions from $\Phi_{6} \rightarrow \Phi_{10}, \Phi_{8} \rightarrow \Phi_{10}$ and $\Phi_{5} \rightarrow \Phi_{10}$, and $\Phi_{4} \rightarrow \Phi_{10}$, $\Phi_{8} \rightarrow \Phi_{10}$ and $\Phi_{3} \rightarrow \Phi_{10}$, respectively. 

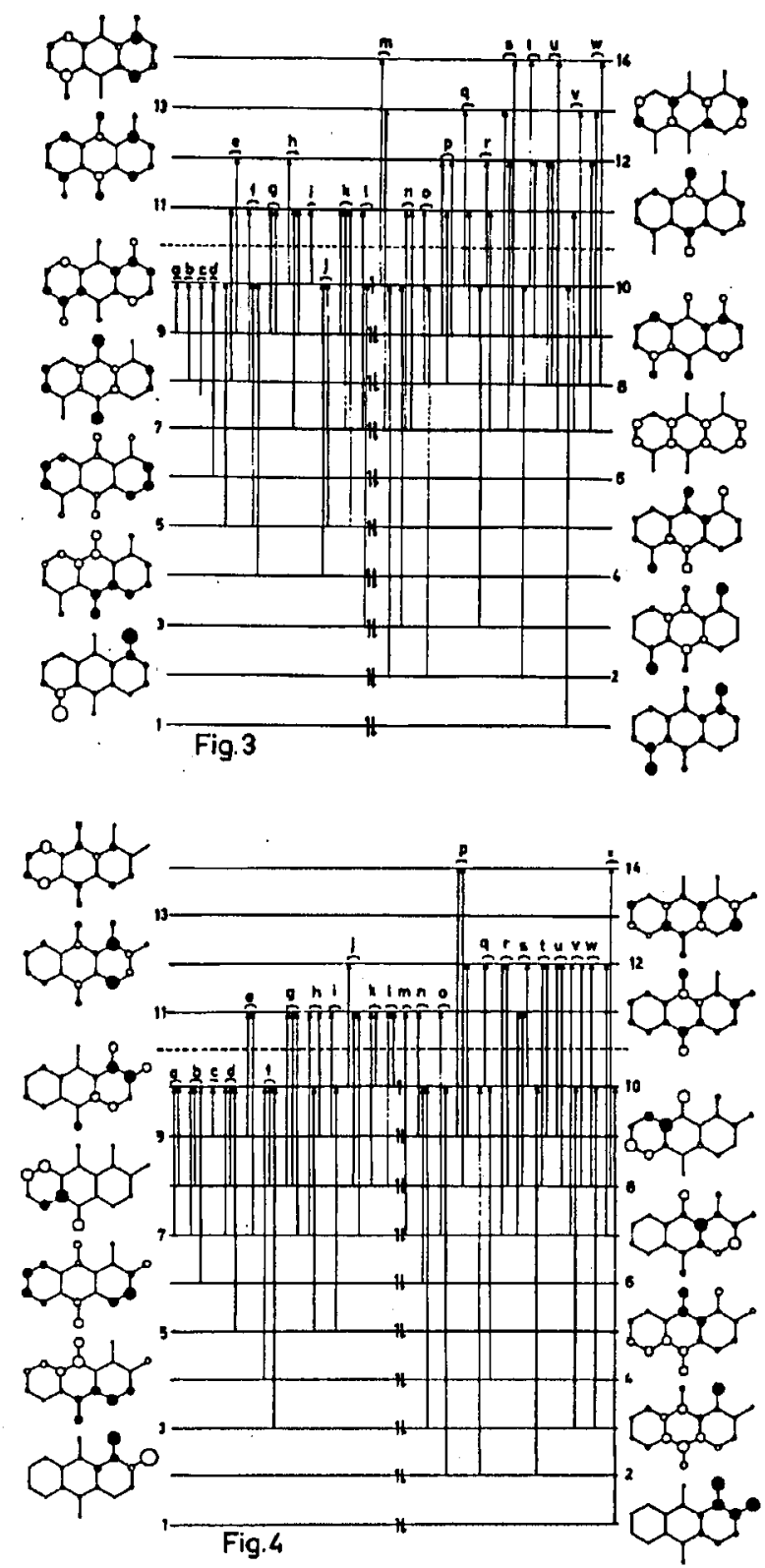

Fig. 3. MO diagram for 1.5-dihydroxy 9.10-anthraquinone cation and its different modes of excitation. The letters $a, b, c$ etc. correspond to the transitions in Table III. For other details, see caption to Fig. 1

Fig. 4 MO diagram for 1.2-dihydroxy 9.10-anthraquinone cation and its different modes of excitation. The letters $a, b, c$ etc. correspond to the transitions in Table IV. For other details, see caption to Fig. 1. 
TABLE III

Results of open-shell SCF-CI calculations for 1.5-dihydroxy 9.10-anthraquinone cation.

\begin{tabular}{|c|c|c|c|c|c|c|}
\hline & $E[\mathrm{eV}]$ & $f^{*}$ & Class & \multicolumn{3}{|c|}{ Character ${ }^{*}$} \\
\hline$a$ & 0.10 & 0.022 & I & $-0.96(9 \rightarrow 10) I$ & & \\
\hline$b$ & 0.62 & 0.000 & I & $0.97(8 \rightarrow 10) \mathrm{I}$ & & \\
\hline$c$ & 0.87 & 0.005 & I & $0.98(7 \rightarrow 10) I$ & & \\
\hline$d$ & 1.96 & 0.000 & I & $-0.85(6 \rightarrow 10) I$ & & \\
\hline$e$ & 2.18 & 0.061 & IB & $-0.62(5 \rightarrow 10) \mathrm{I}$ & $0.49(8 \rightarrow 11) B_{3}$ & $-0.34(9 \rightarrow 12) \mathrm{B}_{3}$ \\
\hline$f$ & 2.44 & 0.012 & $\mathrm{BI}$ & $-0.66(8 \rightarrow 11) \mathrm{B}_{3}$ & $-0.48(5 \rightarrow 10) \mathrm{I}$ & $-0.40(4 \rightarrow 10) \mathbf{I}$ \\
\hline$g$ & 2.62 & 0.000 & B & $0.60(9 \rightarrow 11) \mathrm{B}_{2}$ & $0.60(9 \rightarrow 11) \mathrm{B}_{3}$ & \\
\hline$h$ & 3.27 & 0.000 & B & $-0.48(10 \rightarrow 12) \mathrm{B}_{1}$ & $-0.45(7 \rightarrow 11) \mathrm{B}_{3}$ & $-0.42(9 \rightarrow 11) \mathrm{B}_{2}$ \\
\hline$i$ & 3.32 & 0.125 & $\mathbf{A}$ & $0.85(10 \rightarrow 11) \mathrm{A}$ & & \\
\hline$j$ & 3.48 & 0.132 & I & $0.78(4 \rightarrow 10) \mathrm{I}$ & $-0.47(5 \rightarrow 10) I$ & \\
\hline$k$ & 3.49 & 0.000 & $\mathbf{B}$ & $-0.50(9 \rightarrow 11) \mathrm{B}_{2}$ & $0.42(7 \rightarrow 11) \mathrm{B}_{3}$ & $-0.35(5 \rightarrow 11) \mathrm{B}_{3}$ \\
\hline$l$ & 3.87 & 0.000 & BI & $-0.81(7 \rightarrow 11) \mathrm{B}_{2}$ & $-0.45(3 \rightarrow 10) \mathrm{I}$ & \\
\hline$m$ & 4.09 & 0.001 & BI & $-0.52(10 \rightarrow 14) \mathbf{B}_{1}$ & $-0.39(7 \rightarrow 13) B_{3}$, & $-0.39(2 \rightarrow 10) \mathrm{I}$ \\
\hline$n$ & 4.23 & 0.000 & $\mathrm{BI}$ & $-0.53(3 \rightarrow 10) \mathrm{I}$ & $0.45(7 \rightarrow 11) B_{2}$ & $0.41(7 \rightarrow 11) \mathrm{B}_{3}$ \\
\hline 0 & 4.43 & 0.182 & $\mathrm{BI}$ & $0.77(8 \rightarrow 11) \mathrm{B}_{2}$ & $0.30(2 \rightarrow 10) I$ & \\
\hline$p$ & 4.48 & 0.233 & $\mathrm{~B}$ & $0.75(9 \rightarrow 12) \mathbf{B}_{2}$ & $-0.37(8 \rightarrow 11) B_{2}$ & $0.34(9 \rightarrow 12) \mathrm{B}_{3}$ \\
\hline$q$ & 4.48 & 0.000 & B & $-0.64(10 \rightarrow 13) \mathrm{B}_{1}$ & $0.34(9 \rightarrow 11) \mathrm{B}_{3}$ & \\
\hline$r$ & 4.64 & 0.000 & $\mathrm{BI}$ & $0.54(3 \rightarrow 10) \mathrm{I}$ & $-0.53(10 \rightarrow 12) \mathrm{B}_{1}$ & $0.37(7 \rightarrow 11) \mathrm{B}_{3}$ \\
\hline$s$ & 4.72 & 0.077 & B & $-0.66(9 \rightarrow 13) \mathrm{B}_{2}$ & $-0.40(7 \rightarrow 12) \mathrm{B}_{2}$ & $-0.33(8 \rightarrow 14) \mathrm{B}_{2}$ \\
\hline$t$ & 5.00 & 0.565 & $\mathrm{BI}$ & $0.51(2 \rightarrow 10) \mathrm{I}$ & $-0.43(10 \rightarrow 14) \mathrm{B}_{1}$ & $-0.40(9 \rightarrow 12) \mathrm{B}_{2}$ \\
\hline$u$ & 5.10 & 0.000 & B & $-0.58(8 \rightarrow 12) B_{2}$ & $0.44(8 \rightarrow 12) \mathrm{B}_{3}$ & $0.41(7 \rightarrow 14) \mathrm{B}_{3}$ \\
\hline$v$ & 5.33 & 0.000 & IB & $-0.70(1 \rightarrow 10) \mathrm{I}$ & $0.38(7 \rightarrow 11) B_{3}$ & $-0.35(8 \rightarrow 13) \mathrm{B}_{3}$ \\
\hline$w$ & 5.35 & 0.318 & $\mathrm{~B}$ & $0.59(7 \rightarrow 12) \mathrm{B}_{3}$ & $0.51(9 \rightarrow 13) \mathrm{B}_{2}$ & $0.41(8-14) \mathrm{B}_{3}$ \\
\hline$x$ & 5.61 & 0.306 & IB & $-0.58(2 \rightarrow 10) \mathrm{I}$ & $0.38(6 \rightarrow 11) \mathrm{B}_{3}$ & $0.33(7 \rightarrow 13) \mathrm{B}_{3}$ \\
\hline$y$ & 5.62 & 0.000 & B & $0.85(9 \rightarrow 14) \mathrm{B}_{2}$ & $-0.33(8 \rightarrow 13) \mathrm{B}_{2}$ & \\
\hline$z$ & 5.69 & 0.000 & B & $0.60(8 \rightarrow 12) \mathrm{B}_{2}$ & $0.50(8 \rightarrow 12) \mathrm{B}_{3}$ & $0.33(7 \rightarrow 14) \mathrm{B}_{3}$ \\
\hline
\end{tabular}

Two IB-type transitions are predicted at 4.21 and $4.88 \mathrm{eV}$. The calculations also predict an AI transition with major contributions from $\Phi_{10} \rightarrow \Phi_{11}, \Phi_{5} \rightarrow \Phi_{10}$, and $\Phi_{9} \rightarrow \Phi_{11}$. The transitions $k$ and $l$ arising due to the mixing of configurations $\Phi_{8} \rightarrow \Phi_{11}$ and $\Phi_{10} \rightarrow \Phi_{11}$, are of BA type; the former having a relatively large oscillator strength. All other transitions are of B type.

\subsection{2,5.8-tetrahydroxy 9.10-anthraquinone cation [1.2,5.8-THAQ $\left.Q^{+}\right]$}

The results of MO calculations for electronic states of 1.2,5.8-THAQ cation are presented in Table $\mathrm{V}$ and its MO diagram is shown in Fig. 5. The first three transitions $a, b$, and $c$ are pure I-type one-electron excitations. The next two transitions $d$ and $e$ are also of I-type which arise due to mixing between the configurations $\Phi_{8} \rightarrow \Phi_{12}$ and $\Phi_{7} \rightarrow \Phi_{12}$. Other I-type transitions are $k$ and $v$. The electronic transitions $f, g$, and $h$ result from the mixing of $\Phi_{11} \rightarrow \Phi_{13}$ and $\Phi_{12} \rightarrow \Phi_{13}$. The first two of these transitions are of BA type and the third one is of AB type with their oscillator strengths increasing in the same way as their energies. The transitions $r$ and $s$ are due to the strong interaction of configurations $\Phi_{5} \rightarrow \Phi_{12}$ and $\Phi_{11} \rightarrow \Phi_{15}$ and are assigned as IB and BI, respectively. The transitions $w$ and $x$ are also of BI type which result from the mixing of $\Phi_{11} \rightarrow \Phi_{16}$ and $\Phi_{4} \rightarrow \Phi_{12}$. Among all the transitions given in the table, the most intense one is $t$ and is located at $4.98 \mathrm{eV}$. 
TABLE IV

Results of open-shell SCF-CI calculations for 1.2-dihydroxy 9.10-anthraquinone cation.

\begin{tabular}{|c|c|c|c|c|c|c|}
\hline & $E[\mathrm{eV}]$ & $f^{*}$ & Class & & Character ${ }^{*}$ & \\
\hline$a$ & 0.56 & 0.009 & $\mathrm{I}$ & $-0.65(7 \rightarrow 10) \mathrm{I}$ & $-0.58(8 \rightarrow 10) \mathrm{I}$ & \\
\hline$b$ & 0.94 & 0.004 & I & $0.69(7 \rightarrow 10) \mathrm{I}$ & $-0.48(8 \rightarrow 10) \mathbf{I}$ & $-0.40(6 \rightarrow 10) \mathbf{I}$ \\
\hline$c$ & 1.41 & 0.100 & I & $0.91(9 \rightarrow 10) \mathrm{I}$ & & \\
\hline$d$ & 2.05 & 0.037 & I & $0.60(6 \rightarrow 10) \mathrm{I}$ & $-0.43(8 \rightarrow 10) I$ & $0.41(5 \rightarrow 10) \mathrm{I}$ \\
\hline$e$ & 2.43 & 0.019 & B & $0.63(9 \rightarrow 11) \mathrm{B}_{3}$ & $0.30(7 \rightarrow 11) \mathrm{B}_{3}$ & \\
\hline$f$ & 3.00 & 0.007 & I & $0.57(4 \rightarrow 10) \mathrm{I}$ & $0.34(8 \rightarrow 10) \mathrm{I}$ & $-0.31(3 \rightarrow 10) \mathrm{I}$ \\
\hline$g$ & 3.25 & 0.042 & B & $-0.50(8 \rightarrow 11) B_{3}$ & $0.43(8 \rightarrow 11) B_{2}$ & $0.40(7 \rightarrow 11) \mathrm{B}_{3}$ \\
\hline$h$ & 3.44 & 0.099 & A & $-0.42(10 \rightarrow 11) \mathrm{A}$ & $0.40(5 \rightarrow 10) \mathrm{I}$ & $0.36(9 \rightarrow 11) \mathrm{B}_{2}$ \\
\hline$\because$ & 3.59 & 0.126 & BI & $0.75(9 \rightarrow 11) B_{2}$ & $-0.39(5 \rightarrow 10) \mathrm{I}$ & \\
\hline$j$ & 3.65 & 0.020 & B & $-0.44(10 \rightarrow 12) \mathrm{B}_{1}$ & $0.41(8 \rightarrow 11) B_{3}$ & $0.31(7 \rightarrow 11) \mathrm{B}_{3}$ \\
\hline$k$ & 3.68 & 0.148 & BA & $-0.55(8 \rightarrow 11) \mathrm{B}_{2}$ & $-0.29(10 \rightarrow 11) A$ & \\
\hline$l$ & 3.88 & 0.005 & BA & $0.45(8 \rightarrow 11) \mathrm{B}_{2}$ & $-0.44(10 \rightarrow 11) \mathrm{A}$ & \\
\hline$m$ & 4.17 & 0.067 & B & $0.39(7-11) \mathrm{B}_{2}$ & & \\
\hline$n$ & 4.21 & 0.028 & IB & $0.40(9 \rightarrow 11) \mathrm{B}_{3}$ & $-0.34(6 \rightarrow 10) \mathrm{I}$ & $-0.32(3 \rightarrow 10) \mathrm{I}$ \\
\hline 0 & 4.52 & 0.208 & BI & $0.59(7 \rightarrow 11) B_{2}$ & $-0.35(2 \rightarrow 10) I$ & \\
\hline$p$ & 4.73 & 0.024 & $\mathbf{B}$ & $0.50(9 \rightarrow 14) \mathrm{B}_{3}$ & $0.42(8 \rightarrow 14) \mathrm{B}_{3}$ & $-0.31(9 \rightarrow 12) \mathrm{B}_{3}$ \\
\hline$q$ & 4.88 & 0.163 & IB & $0.54(2 \rightarrow 10) \mathrm{I}$ & $-0.41(10 \rightarrow 12) \mathrm{B}_{1}$ & $-0.30(4 \rightarrow 10) \mathbf{I}$ \\
\hline$r$ & 4.97 & 0.363 & B & $0.57(9 \rightarrow 12) B_{2}$ & $0.36(8 \rightarrow 12) B_{2}$ & \\
\hline$s$ & 5.02 & 0.213 & B & $0.39(7 \rightarrow 11) B_{3}$ & $-0.36(8 \rightarrow 12) \mathrm{B}_{2}$ & $0.36(10 \rightarrow 12) \mathrm{B}_{1}$ \\
\hline$t$ & 5.12 & 0.049 & BI & $-0.41(2 \rightarrow 10) \mathrm{I}$ & $-0.41(8 \rightarrow 12) \mathrm{B}_{2}$ & $0.34(9 \rightarrow 12) \mathrm{B}_{2}$ \\
\hline$u$ & 5.16 & 0.053 & B & $-0.53(9 \rightarrow 12) \mathrm{B}_{2}$ & $0.34(8 \rightarrow 12) \mathrm{B}_{2}$ & \\
\hline$v$ & 5.28 & 0.194 & BI & $-0.40(7 \rightarrow 12) \mathrm{B}_{2}$ & $-0.37(3 \rightarrow 10) \mathrm{I}$ & $-0.36(8 \rightarrow 12) \mathrm{B}_{2}$ \\
\hline$w$ & 5.39 & 0.057 & $\mathrm{BI}$ & $-0.37(8 \rightarrow 12) \mathrm{B}_{2}$ & $0.34(3 \rightarrow 10) \mathrm{I}$ & \\
\hline$x$ & 5.47 & 0.200 & $\mathrm{BI}$ & $-0.33(7 \rightarrow 12) \mathrm{B}_{2}$ & $0.31(8 \rightarrow 14) B_{2}$ & $0.30(1 \rightarrow 10) I$ \\
\hline$y$ & 5.70 & 0.081 & B & $0.75(6 \rightarrow 11) \mathrm{B}_{2}$ & $-0.38(8 \rightarrow 13) \mathrm{B}_{2}$ & \\
\hline$z$ & 5.80 & 0.047 & IB & $-0.48(1 \rightarrow 10) \mathrm{I}$ & $-0.37(10 \rightarrow 13) \mathrm{B}_{1}$ & $0.37(6 \rightarrow 11) \mathrm{B}_{2}$ \\
\hline
\end{tabular}

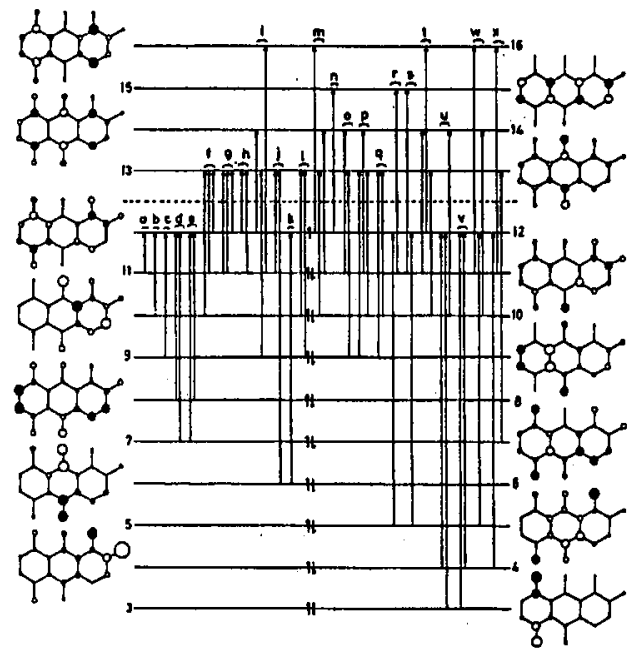

Fig. 5. MO diagram for 1.2,5.8-tetrahydroxy 9.10-anthraquinone cation and its different modes of excitation. The letters $a, b, c$ etc. correspond to the transitions in Table V. For other details, see caption to Fig. 1. 
TABLE V

Results of open-shell SCF-CI calculations for 1.2,5.8-tetrahydroxy 9.10-anthraquinone cation.

\begin{tabular}{|c|c|c|c|c|c|c|}
\hline & $E[\mathrm{eV}]$ & $f^{*}$ & Class & & Character $^{*}$ & . \\
\hline$a$ & 0.27 & 0.046 & I & $-0.93(11 \rightarrow 12) \mathrm{I}$ & & \\
\hline$b$ & 0.90 & 0.002 & I & $0.95(10 \rightarrow 12) \mathrm{I}$ & & \\
\hline$c$ & 1.22 & 0.001 & I & $-0.97(9 \rightarrow 12) \mathrm{I}$ & & \\
\hline$d$ & 2.00 & 0.054 & I & $0.75(8 \rightarrow 12) \mathrm{I}$ & $-0.44(7 \rightarrow 12) \mathrm{I}$ & \\
\hline$e$ & 2.43 & 0.023 & I & $0.67(7 \rightarrow 12) \mathrm{I}$ & $0.38(8 \rightarrow 12) I$ & \\
\hline$f$ & 2.50 & 0.000 & $\mathbf{B A}$ & $-0.54(10 \rightarrow 13) \mathrm{B}_{3}$ & $0.37(11 \rightarrow 13) \mathrm{B}_{3}$ & $-0.34(12 \rightarrow 13) \mathrm{A}$ \\
\hline$g$ & 2.61 & 0.073 & BA & $0.57(11 \rightarrow 13) \mathrm{B}_{2}$ & $0.52(11 \rightarrow 13) \mathrm{B}_{3}$ & $0.34(12 \rightarrow 13) \mathrm{A}$ \\
\hline$h$ & 3.08 & 0.154 & $\mathrm{AB}$ & $0.74(12 \rightarrow 13) A$ & $-0.37(11 \rightarrow 13) \mathrm{B}_{2}$ & \\
\hline$i$ & 3.33 & 0.008 & B & $-0.49(12 \rightarrow 14) \mathrm{B}_{1}$ & $0.47(9 \rightarrow 13) \mathrm{B}_{3}$ & $0.30(11 \rightarrow 16) \mathrm{B}_{3}$ \\
\hline$j$ & 3.38 & 0.082 & B & $-0.54(11 \rightarrow 13) \mathrm{B}_{2}$ & $0.36(6 \rightarrow 13) \mathrm{B}_{3}$ & \\
\hline$k$ & 3.76 & 0.051 & I & $-0.79(6 \rightarrow 12) \mathbf{I}$ & & - \\
\hline$l$ & 3.84 & 0.022 & $\mathrm{~B}$ & $0.58(10 \rightarrow 13) B_{2}$ & $0.58(9 \rightarrow 13) \mathrm{B}_{2}$ & \\
\hline$m$ & 4.08 & 0.017 & B & $-0.56(12 \rightarrow 16) \mathrm{B}_{1}$ & $-0.39(10 \rightarrow 13) \mathrm{B}_{3}$ & $0.35(11 \rightarrow 14) \mathrm{B}_{3}$ \\
\hline$n$ & 4.21 & 0.010 & $\mathbf{B}$ & $-0.57(12 \rightarrow 15) \mathrm{B}_{1}$ & & \\
\hline$o$ & 4.34 & 0.102 & $\mathrm{~B}$ & $0.62(11 \rightarrow 14) \mathrm{B}_{2}$ & $-0.36(9 \rightarrow 13) \mathrm{B}_{2}$ & \\
\hline$p$ & 4.50 & 0.018 & B & $-0.44(9 \rightarrow 13) \mathrm{B}_{3}$ & $-0.41(11 \rightarrow 14) B_{2}$ & $0.36(10 \rightarrow 13) \mathrm{B}_{2}$ \\
\hline$q$ & 4.58 & 0.280 & $\mathrm{~B}$ & $0.37(9 \rightarrow 13) \mathrm{B}_{2}$ & $-0.33(10 \rightarrow 13) \mathrm{B}_{2}$ & \\
\hline$r$ & 4.70 & 0.042 & IB & $-0.66(5 \rightarrow 12) \mathrm{I}$ & $0.35(11 \rightarrow 15) \mathrm{B}_{2}$ & \\
\hline$s$ & 4.79 & 0.028 & $\mathrm{BI}$ & $0.63(11 \rightarrow 15) \mathrm{B}_{2}$ & $0.46(5 \rightarrow 12) \mathrm{I}$ & \\
\hline$t$ & 4.98 & 0.544 & B & $-0.38(11 \rightarrow 14) \mathrm{B}_{2}$ & $-0.36(12 \rightarrow 16) \mathrm{B}_{1}$ & $0.31(10 \rightarrow 13) \mathrm{B}_{2}$ \\
\hline$u$ & 5.08 & 0.123 & IB & $-0.41(4 \rightarrow 12) \mathrm{I}$ & $0.41(3 \rightarrow 12) \mathrm{I}$ & $-0.37(10 \rightarrow 14) \mathrm{B}_{3}$ \\
\hline$v$ & 5.11 & 0.025 & $\mathbf{I}$ & $0.70(3 \rightarrow 12) \mathrm{I}$ & $0.35(4 \rightarrow 12) \mathrm{I}$ & \\
\hline$w$ & 5.31 & 0.058 & BI & $-0.46(11 \rightarrow 16) \mathrm{B}_{2}$, & $-0.42(4 \rightarrow 12) \mathrm{I}$ & $0.37(10 \rightarrow 14) \mathrm{B}_{3}$ \\
\hline$x$ & 5.35 & 0.089 & BI & $-0.41(4 \rightarrow 12) \mathrm{I}$ & $0.39(11 \rightarrow 16) \mathrm{B}_{2}$ & $-0.36(7 \rightarrow 13) \mathrm{B}_{2}$ \\
\hline$y$ & 5.41 & 0.213 & B & $0.42(9 \rightarrow 14) \mathrm{B}_{2}$ & $-0.37(10 \rightarrow 14) \mathrm{B}_{2}$ & $-0.35(9 \rightarrow 14) \mathrm{B}_{3}$ \\
\hline$z$ & 5.53 & 0.137 & B & $0.78(8 \rightarrow 13) \mathrm{B}_{2}$ & & \\
\hline
\end{tabular}

\subsection{Correlation of electronic transitions}

In order to understand the behaviour of electronic transitions in moving from one system to another, we have shown in Fig. 6 a correlation diagram for the calculated transition energies and intensities of the monopositive ions of dihydroxyand tetrahydroxy-anthraquinones. For this, we restrict our discussion to energies up to $5 \mathrm{eV}$ only as these are mostly the lower-energy transitions that characterize radical ions. Since the electronic transitions in the energy range $3-5 \mathrm{eV}$ are overcrowded, we have omitted in the diagram the transitions with low oscillator strengths.

The first three transitions in these systems are dominantly of I-type having low oscillator strengths except for the third one in 1.2-DHAQ which is located at $1.41 \mathrm{eV}$ and has a moderate $f$-value. The first two transitions in 1.4-DHAQ cation arise due to contributions from I and B type configurations, whereas in all the remaining cases these transitions are of pure I type. There is a general decreasing trend in the energies of these transition in moving from (a) to (e) except for the case (c) where they are slightly blue-shifted. The fourth transition in 1.4-DHAQ, 1.2,5.8-THAQ and 1.2-DHAQ cations is pure I type, whereas in the remaining two systems it is mixed (IB type) arising from the interaction of I and B type configurations. All these transitions have moderate oscillator strengths and their energies increase gradually in moving from 1.4-DHAQ to 1.8-DHAQ cation. 


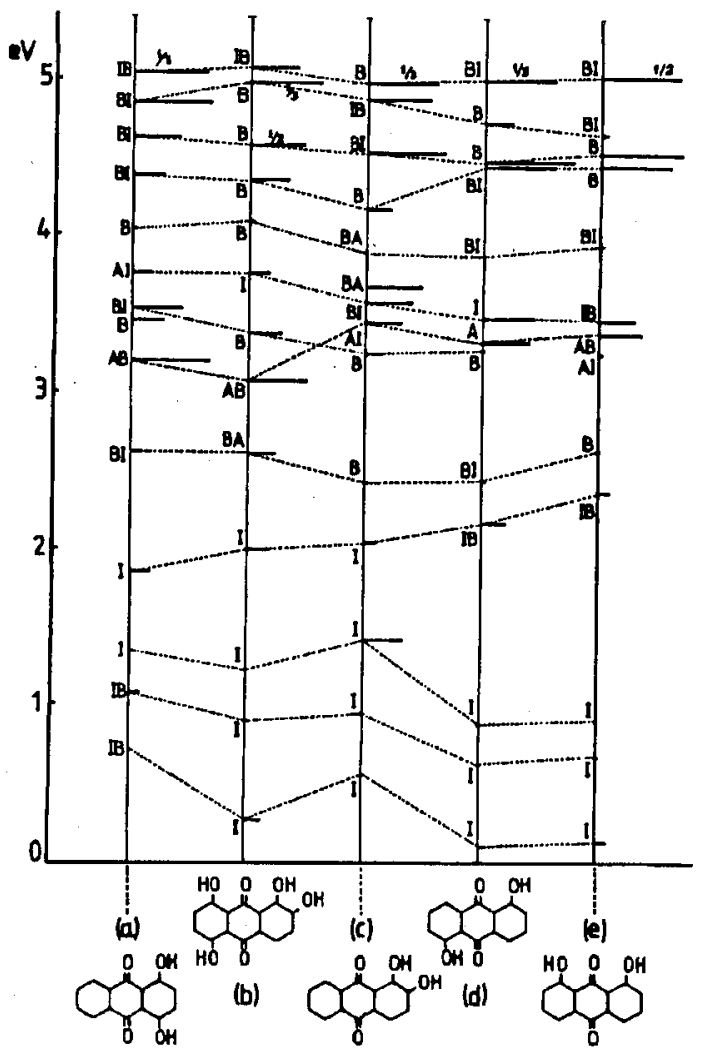

Fig. 6. Correlation diagram for the calculated electronic transitions of radical cations of (a) 1.4-dihydroxy 9.10-anthraquinone (1.4-DHAQ), (b) 1.2,5.8-tetrahydroxy 9.10-anthraquinone (1.2,5.8-THAQ), (c) 1.2- dihydroxy 9.10-anthraquinone (1.2-DHAQ), (d) 1.5-dihydroxy 9.10-anthraquinone (1.5-DHAQ), and (e) 1.8-dihydroxy 9.10-anthraquinone (1.8-DHAQ). The letters I, A, and B indicate the classes to which electronic transitions belong. The horizontal solid lines give the measure of the calculated oscillator strengths $f$ of the transitions and the fractional numbers show the amount by which the $f$-values have been reduced in the figure.

The next transition in these ionic systems is of $B$ type which is either pure or mixed with I or A type configurations. In all the cases, except for 1.2,5.8-TIAQ, this transition has low oscillator strength. However, it does not show any significant energy shift in moving from (a) to (e). One of the important transitions in radical cations is that of A type belonging to the non-Koopmans class. In the molecular ions under consideration, it is often mixed with B or I type configurations and has sufficiently large oscillator strength in all the systems. At first, the energy of this transition shows a slight decrease, but it gets appreciably blue-shifted for the third case and remains almost uniform in the rest of the systems.

In addition to the lower-energy I transitions, there is yet another I type transition which is located in the energy interval $3-4 \mathrm{eV}$ and is often mixed with $\mathrm{A}$ or 
I type configurations. Except for the case (b), this transition is slightly red-shifted and has appreciable oscillator strength. The remaining transitions are predominantly of B type. It has been possible to correlate most of them on the basis of their energies and intensities. It is noticed that these transitions generally do not show any significant shift in their energy in moving from one system to another.

\subsection{Estimation of the first ionizalion potentials of hydroxy-substituted anthraquinones}

In a recent paper [2], it was demonstrated that the first IPs of condensed-ring aromatic hydrocarbons can be estimated from the lowest-energy non-Koopmans state of their radical cations using the correlation

$$
\begin{aligned}
& \mathrm{IP}_{1}^{\mathrm{cal}}=5.35+0.43 E_{\mathrm{A}}^{\mathrm{SCF}-\mathrm{CI}}+16.43 / N \\
& \text { S.E. }\left(\mathrm{IP}_{1}\right)=0.12 \mathrm{eV}
\end{aligned}
$$

where $N$ is the total number of $\pi$-atomic centres in a molecule and $E_{\mathrm{A}}^{\mathrm{SCF}-\mathrm{CI}}$ is the energy of the lowest non-Koopmans state as calculated from the open-shell SCF-CI theory with limited CI. The first IPs calculated using Eq. (4) are listed in Table VI and are compared with the observed IPs for 1.4-DIIAQ and 1.8-DHAQ. The predictions of the above relation for 1- and 2-naphthols were in close agreement with experiment [2], but for the dihydroxy-anthraquinones under investigation, the discrepancy is surprisingly large. Such a large variation in the calculated and observed IPs is difficult to understand as sufficient experimental data are not yet available to judge the accuracy of the results.

TABLE VI

Estimated first ionization potentials of hydroxy-substituted anthraquinones obtained from Eq. (4) using open-shell SCF-CI energies of the lowest-energy

\begin{tabular}{|c|c|c|c|c|}
\hline Substituted hydrocarbons & $N^{*}$ & $E_{\mathrm{A}}^{\mathrm{SCF}-\mathrm{CI}}$ & $1 \mathrm{P}_{1}^{\mathrm{cal} * *}$ & IIPobs*** \\
\hline 1.4-dihydroxy 9.10 -anthraquinone & 18 & 3.20 & 7.63 & 8.2 \\
\hline 1.8-dihydroxy 9.10 -anthraquinone & 18 & 3.38 & 7.71 & 8.8 \\
\hline 1.5-dihydroxy 9.10 -anthraquinone & 18 & 3.32 & 7.70 & - \\
\hline 1.2-dihydroxy 9.10 -anthraquinone & 18 & 3.44 & 7.74 & - \\
\hline 1.2,5.8-tetrahydroxy 9.10 -anthraquinone & 20 & 3.08 & 7.50 & - \\
\hline
\end{tabular}
non-Koopmans A-type transitions for their cations.

\section{Conclusions}

The open-shell SCF calculations with limited CI provide useful information about electronic transitions of radical cations of hydroxy-substituted anthraquinones. The correlation diagram is particularly interesting as it reveals the 
true behaviour of the transitions in moving from one system to another and shows the shifts in energies and intensities with the substitution of hydroxyl groups at different atomic positions in these molecular ions. The calculated energy of the lowest non-Koopmans states of the substituted anthraquinone cations does provide a simple and useful parameter to estimate the first ionization potentials of their neutral precursors. However, to judge the predictability of the IP $/ E_{\mathrm{A}}$ correlation, more experimental data, both for ionization potentials as well as for electronic states on radical cations are required. Monopositive ions of hydroxy-anthraquinones can, in principle, be produced in matrices like polymers which have already been found to be very suitable for incorporating polycyclic aromatic hydrocarbons. Our experimental work in this direction is in progress.

\section{References}

[1] Z.H. Khan, Z.U. Khan, Z.H. Zaidi, Can. J. Spectrosc. 33, 170 (1988).

[2] Z.H. Khan, Acta Phys. Pol. A 80, 29 (1991).

[3] Z.H. Khan, Int. J. Quant. Chem. 42, 1717 (1992).

[4] W.A. Remers, The Chemistry of Anti-Tumour Antibiotics, Vol. 1, Wiley, New York 1979.

[5] B. Keita, I. Kawenoky, J. Kossany, D. Garreau, L. Nadio, J. Electroanal. Chem. 145, 293 (1983).

[6] D. Margetic, M. Eckert-Maksic, Z.B. Maksic, J. Mol. Struct. (Theochem.) 277, 161 (1992).

[7] A.D. Broadbent, W.D. Hewson, H.A. McDonald, R.J. Melanson, Can. J. Chem. 55, 2946 (1977).

[8] H.C. Longuet-Higgins, J.A. Pople, Proc. Phys. Soc. A 68, 591 (1955).

[9] J. Wasilewski, Acta Phys. Pol. A 38, 349 (1970).

[10] N. Mataga, K. Nishimoto, Z. Phys. Chem. Frankfurt (Main) 13, 140 (1957).

[11] Z.H. Khan, Spectrochim. Acta A 46, I (1990).

[12] S. Millefiori, A. Millefiori, Spectrochim. Acta A 44, 17 (1988). 